\title{
Questionário sobre qualidade de vida de atletas: adaptação e evidências de validade para bailarinos
}

\author{
Encuesta sobre la calidad de vida de los atletas: adaptación \\ y evidencias de validez para bailarines \\ Survey on Quality of Life of Athletes: Adaptation \\ and Ualidity Euidences for Dancers
}

\author{
Andressa Melina Becker da Silva* \\ Curso de Psicologia e Educação Física da Universidade de Sorocaba, Sorocaba-SP, Brasil. \\ Sônia Regina Fiorim Enumo** \\ Renan de Morais Afonso** \\ Murilo Fernandes de Araújo** \\ Tatiane Stephan Rocchatti Luz ${ }^{* *}$ \\ Programa de Pós Graduação em Psicologia da Pontifícia Universidade Católica de Campinas, Campinas-SP. \\ Lucas de Francisco Carvalho*** \\ Programa de Pós Graduação em Psicologia da Universidade São Francisco, Itatiba-SP. \\ Isabella Goulart Bittencourt**** \\ Programa de Pós Graduação em Psicologia da Universidade Federal de Santa Catarina, Florianópolis-SC.
}

Doi: http://dx.doi.org/10.12804/revistas.urosario.edu.co/apl/a.4575

\section{Resumo}

A qualidade de vida consiste em um construto multidimensional composto por dimensões cognitivas, emocionais e comportamentais, de socialização e percepção de saúde. O objetivo deste estudo foi verificar as evidências de validade do Questionário de Qua- lidade de Vida sobre Atletas (QQVA) aplicando-o em bailarinos adolescentes (QQVA-B). Foram avaliados 313 bailarinos, com idade entre 10 e 19 anos $(M=15.56$; \pm 3.59), durante festivais de dança no Brasil de caráter nacional e internacional. Foi realizada a verificação das evidências de validade de conteúdo (avaliação de especialistas para pertinências teórica e prática, com

\footnotetext{
* Curso de Psicologia e Educação Física da Universidade de Sorocaba, Sorocaba-SP, Brasil.

** Programa de Pós Graduação em Psicologia da Pontifícia Universidade Católica de Campinas, Campinas-SP.

*** Programa de Pós Graduação em Psicologia da Universidade São Francisco, Itatiba-SP.

****Programa de Pós Graduação em Psicologia da Universidade Federal de Santa Catarina, Florianópolis-SC.
}

Para citar este artigo: da Silva, A. M. B., Enumo, S. R. F., Afonso, R. de M., de Araújo, M. F., Luz, T. S. R., Carvalho, L. de F., \& Bittencourt, I. G. (2019). Questionário sobre qualidade de vida de atletas: adaptação e evidências de validade para bailarinos. Avances en Psicología Latinoamericana, 37(1), 121-131. Doi: http://dx.doi.org/10.12804/revistas.urosario.edu.co/ apl/a.4575 
adaptações para bailarinos e linguagem - acessível para adolescentes), além da verificação das evidências de validade da consistência interna, com análise fatorial exploratória e confirmatória. Os resultados, discutidos ao longo do artigo, indicam que o instrumento apresenta propriedades psicométricas satisfatórias para ser utilizado em bailarinos.

Palavras-chave: bailarinos, qualidade de vida, psicologia do esporte, testes psicológicos.

\section{Resumen}

La calidad de vida es un constructor multidimensional e incluye dimensiones cognitiva, emocional y comportamental, de socialización y percepción de la salud. El objetivo de este estudio fue evaluar la evidencia de validez de la escala Calidad de Vida para los Atletas (QQVA) aplicándola en bailarines adolescentes (QQVA-B). Se evaluaron 313 bailarines, con edades entre 10 y 19 años $(M=15.56 ; \pm 3.59)$ durante los festivales de danza en Brasil, en nivel nacionales e internacionales. Se realizó la verificación de las pruebas de la validez de contenido (la evaluación de expertos con la pertinencia teórica y práctica, con adaptaciones para los bailarines y lenguaje accesible a los adolescentes) y de la validez de las pruebas de consistencia interna, con un análisis factorial exploratorio y confirmatorio. Los resultados discutidos en todo el artículo, indican que el instrumento tiene propiedades psicométricas satisfactorias para su uso en los bailarines.

Palabras clave: bailarines, calidad de vida, psicología del deporte, pruebas psicológicas.

\section{fibstract}

The quality of life is a multidimensional construct and includes cognitive, emotional and behavioral, socialization and health perception dimensions. The aim of this study was to verify evidences of validity of the Quality of Life Questionnaire for Athletes (QQVA) applying it in adolescent dancers (QQVA-B). We evaluated 313 dancers, aged between 10 and 19 years $(M=15.56 ; \pm 3.59)$ during dance festivals in Brazil, national and international level.
Verification of validity evidences (expert evaluation to theoretical and practical pertinence, with adaptations for dancers and language - accessible to adolescents) was performed, in addition to verifying the validity evidence of internal consistency, with exploratory and confirmatory factor analysis and Cronbach alfa. The results, discussed throughout the article, indicate that the instrument has adequate psychometric properties for its use in dancers.

Keywords: Dancers, quality of life, sport psychology, psychological tests.

\section{Introdução}

A qualidade de vida está relacionada ao grau de satisfação que o indivíduo tem em vários aspectos de sua vida - físicos, psicológicos e socioculturais -, vivenciando-os de forma harmoniosa (Akranaviciute \& Ruzevicius, 2007; Fayers \& Machin, 2013). Para esses autores, a qualidade de vida consiste em um construto multidimensional cujas dimensões são cognitivas, emocionais e psíquicas, de socialização e percepção de saúde. Segundo a Organização Mundial da Saúde (OMS), a qualidade de vida é influenciada, de forma complexa, pela saúde física, estados psicológicos, relações sociais, nível de independência, entre outros (World Health Organization, 1997).

O exercício físico é um dos aspectos que pode influenciar diretamente a qualidade de vida, sendo considerada, por Matsudo et al. (2002), um importante fator para a promoção de saúde. Entretanto, atletas são expostos a cargas extremas de treinamento, causando desgaste físico e mental, prejudicial à saúde, podendo refletir em efeito reverso da prática física (Aksoy \& Aksoy, 2015). Portanto, a análise da qualidade de vida de atletas abrange determinantes: a) objetivos, como a capacidade física para o rendimento esportivo, aspectos antropométricos e biomecânicos, condições climáticas, entre outras, e b) subjetivos, que incluem os interesses, motivações, expectativas, experiências 
próprias, opiniões e preconceitos, sentimentos e emoções. Estes últimos possibilitam a avaliação de cada atleta quanto à percepção subjetiva de quais fatores influenciam a qualidade de vida e em que grau ocorre tal influência (Cunha, 2008).

Embora a dança não possa ser considerada um esporte, é possível fundamentá-la a partir dos princípios da Psicologia do Esporte e do Exercício Físico, pois esta prática exige demandas físicas e psicológicas semelhantes às dos outros esportes, além de também haver competições entre equipes e corpos de baile nos festivais de dança (Silva \& Tkac, 2012; Silveira \& Pinto, 2001). Nesse sentido, toda a demanda exigida nos treinamentos e competições pode influenciar na qualidade de vida dos bailarinos. O presente estudo buscou compreender esse contexto para bailarinos adolescentes. Nesse ponto, é importante mencionar que a adolescência é um período marcado pela passagem da infância para vida adulta, caracterizando-se por intensas mudanças, dúvidas e indecisões (Breinbauer \& Maddaleno, 2008). Esse aspecto pode agravar sua percepção sobre os treinamentos, aulas e ensaios e reduzir sua qualidade de vida. Deve-se, portanto, atentar para a qualidade de vida desses bailarinos adolescentes, considerando que o nível de exigência e treinamento poderá influenciar na saúde física, psíquica e social do sujeito durante esta e outras etapas do desenvolvimento.

Mundialmente, na Psicologia do Esporte, não é difícil encontrar testes válidos para avaliação psicológica em atletas. No Brasil, a validação de instrumentos não é uma prática tão comum, principalmente quando se refere à Psicologia para o Exercício Físico, mais especificadamente para a dança. Nessa área, há uma carência de instrumentos válidos. Até o momento, existe um estudo de validação publicado que se preocupa em mensurar a autoeficácia em bailarinos adolescentes (Silva et al., 2015).

Nesse contexto, muitos profissionais realizam avaliação psicológica em bailarinos utilizando instrumentos válidos para atletas, sem verificar suas propriedades psicométricas e adaptá-los para a realidade da dança, que possui especificidades e que requer atenção (Gonçalves \& Belo, 2007; Silva \& Tkac, 2012). Lima, Silva e Barreto (2014) avaliaram a qualidade de vida e sua relação com as lesões em onze bailarinos, utilizando o SF-36 (The Medical Outcomes Study 36-item Short-Form), questionário validado e genérico de avaliação de saúde, para mensurar a qualidade de vida. Embora seja um instrumento de fácil compreensão, não se destina especificamente à área do esporte e da dança. Outro instrumento utilizado para avaliação da qualidade de vida em atletas é o WHOQOL Bref, desenvolvido pela Organização Mundial de Saúde (Esteves et al., 2015), porém não é específico para atletas e, portanto, apresenta limitações.

Para avaliar os fatores determinantes para a qualidade de vida específica dos atletas, Cunha (2008) construiu e validou o "Questionário sobre Qualidade de Vida de Atletas" (QQVA) para o Brasil. $\mathrm{O}$ instrumento foi baseado no Questionário para Avaliação da Qualidade de Vida [WHOQOL-100] (WHOQOL Group, 1994), proposto pela Organização Mundial de Saúde e destinado a avaliar a qualidade de vida na população em geral. O estudo de validação do QQVA (Cunha, 2008) foi realizado com 298 atletas (179 homens e 119 mulheres) de 14 a 20 anos, das modalidades esportivas: natação, voleibol, basquetebol, futsal, futebol, ciclismo, atletismo, handebol, taekwondo, tênis de campo, esgrima e tiro com arco. Sua escolha se deu pelo fato de ser um instrumento breve, de fácil aplicação, considerando que em meio à correria das atividades diárias os atletas não têm paciência e tempo para preencher questionários longos. A expectativa do autor era que o instrumento se estruturasse em três dimensões - biológica, psicológica e social. Nota-se que, apesar de seu processo de validação ter sido realizado com atletas de diferentes modalidades esportivas, não foram incluídos bailarinos. Desse modo objetivou-se, portanto, verificar as evidências de validade do QQVA para a população de bailarinos adolescentes. 


\section{Método}

\section{Participantes}

Participaram do estudo 313 bailarinos brasileiros, de ambos os sexos (91.37\% mulheres), com idade entre 10 e 19 anos $(M=15.56 ; \pm 3.59)$. Eles dançam, em média, há 8,11 anos ( \pm 3.86$)$, compondo uma amostra de conveniência. A maioria tinha Ensino Médio incompleto (38\%), seguido de Ensino Fundamental incompleto (34\%), Ensino Fundamental completo (11\%), Ensino Médio completo (9\%) e Ensino Superior incompleto (8\%). O nível socioeconômico dos participantes era, em média, classificado como classe $\mathrm{C} 1$ (renda média familiar bruta mensal de 1541.00 reais ou 3513.48 dólares).

\section{Instrumentos}

\section{Questionário de Qualidade de Vida sobre Atletas (QQVA)}

Como dito anteriormente, o instrumento foi validado por Cunha (2008). Nesse processo, alguns aspectos metodológicos foram seguidos. A validade de conteúdo foi feita com oito juízes especialistas na área, obtendo-se coeficientes para a clareza da linguagem $(0.9509)$ e a pertinência prática $(0.9214)$ considerados bons. Os itens apresentaram cargas fatoriais entre 0.754 e 0.898 , e não possuíram cargas fatoriais altas nos outros fatores (menores que 0.280 ). A consistência interna do instrumento, medida pelo Alpha de Cronbach, foi $\alpha=0.733$, considerado válido e fidedigno para avaliação da percepção da qualidade de vida de atletas. Sua versão final é composta por 14 itens agrupados em cinco fatores: a) sinais e sintomas de supertreinamento; b) condições básicas para saúde; c) relacionamento social no ambiente esportivo; d) estados emocionais do atleta; e e) planejamento e periodização do treinamento esportivo.

Por apresentar evidências de validade, o QQVA em sua versão final - 14 questões - foi utilizado no presente estudo. No entanto, o instrumento sofreu adaptações em sua linguagem, para que fosse compreendido por bailarinos adolescentes, considerando a faixa etária e as diferenças de contexto para atletas e bailarinos.

Critério de Classificação Econômica Brasil (Associação Brasileira de Empresas de Pesquisa [ABEP, 2013] <http://www.abep.org/novo/Content. aspx?SectionID=84. É um questionário sobre aspectos socioeconômicos dos participantes para determinar sua classe social. É levantada a quantidade de bens materiais e ambientes da residência. A classificação é dada pela soma de pontos dos itens e sua categorização com base numa linha de corte. Os resultados ser classificados nas classes: A, B1, B2, C1, C2, D, E.A classe "A" tem o maior poder aquisitivo (renda média familiar bruta mensal = 29.471.28 dólares) e a classe E, a de menor poder econômico (renda média familiar bruta mensal = 1087.56 dólares).

\section{Procedimento e Análise de Dados}

O estudo seguiu as normas éticas estabelecidas pela Resolução n ${ }^{\circ}$ 466/2012 do Conselho Nacional de Saúde e foi aprovado pelo Comitê de Ética em Pesquisa da universidade (Parecer $\left.n^{\circ} 770.131\right)$. Os participantes foram convidados a participar da pesquisa durante festivais de dança, de caráter nacional e internacional, realizados no Brasil. Os pais ou responsáveis pelos participantes foram informados sobre os objetivos da pesquisa, metodologia, riscos e benefícios, participando voluntariamente após ler e assinar o Termo de Consentimento Livre e Esclarecido. A aplicação dos instrumentos ocorreu antes dos ensaios de passagem de palco, no dia da competição.

Inicialmente, foi realizada uma revisão do instrumento QQVA para o contexto dos bailarinos, sendo necessário modificar alguns termos, como por exemplo: atleta para bailarino; treinador, equipe técnica para professor, ensaiador; treinamentos para aulas e ensaios; e competições para festivais 
e espetáculos. Após esta adequação, foram adotados alguns procedimentos empíricos. Para a verificação das evidências de validade de conteúdo, seguiram-se as proposições de Pasquali (2004), realizando-se a análise dos itens do instrumento por cinco juízes (dois professores de português, um psicólogo do Esporte, dois educadores físicos com especialidade em dança), para observar a análise semântica, i.e., a compreensão dos itens, e sua pertinência prática e teórica. Para isso, foi utilizado um protocolo adaptado de Balbinotti, Benetti e Terra (2007), que considera a clareza de linguagem, pertinência prática e teórica dos itens do instrumento. Para verificação da clareza de linguagem, foi perguntado aos juízes: "Você acha que estes itens são claros o suficiente e, portanto, serão entendidos pelos bailarinos? Em que extensão?"; para a pertinência prática: "Você acha que estes itens são pertinentes para os bailarinos? Em que extensão?". No espaço para observações, podem ser indicadas falhas, sugerir correções ou mesmo exclusão de determinados itens. Para responder a essas questões, os juízes utilizaram uma escala de cinco pontos: 1 -Pouquíssima, 2 -Pouca, 3 Média, 4 -Muita, 5 - Muitíssima.

Com as respostas dos juízes, realizou-se o cálculo do Coeficiente de Validade do Conteúdo (CVC) para a clareza da linguagem e para a pertinência prática, conforme proposto por Hernández-Nieto (2002):

1) Com base nas notas dos juízes, calcula-se a média das notas de cada item $(\mathrm{Mx})$ :

$\mathrm{Mx}=$ soma das notas dos juízes/ número de juízes que avaliaram o item;

2) Com base na média, calcula-se o CVC para cada item (CVCi):

$\mathrm{CVC}_{\mathrm{i}}=$ média das notas de cada item/ valor máximo que o item poderia receber;

3) Foi realizado o cálculo do erro $\left(\mathrm{P}_{\mathrm{e}}\right)$, para descontar possíveis vieses dos juízes avaliadores, para cada item:
$\mathrm{P}_{\mathrm{ei}}=(1 /$ número de juízes que avaliaram o item $)$ número de juízes que avaliaram o item

4) Com isso, o CVC final de cada item $\left(\mathrm{CVC}_{\mathrm{c}}\right)$ foi assim calculado:

$\mathrm{CVC}_{\mathrm{c}}=\mathrm{CVC}_{\mathrm{i}}-\mathrm{Pe}_{\mathrm{i}}$

5) Para o cálculo do $\mathrm{CVC}$ total do questionário $\left(\mathrm{CVC}_{\mathrm{t}}\right)$, para cada uma das características (clareza de linguagem e pertinência prática), foi usada a fórmula:

$\mathrm{CVC}_{\mathrm{t}}=\mathrm{Mcvc}_{\mathrm{i}}-\mathrm{Mpe}_{\mathrm{i}}$

onde: $\mathrm{Mcvc}_{\mathrm{i}}$ representa a média dos coeficientes de validade de conteúdo dos itens do questionário e $\mathrm{Mpe}_{\mathrm{i}}$, a média dos erros dos itens do questionário.

Para avaliação da dimensão teórica do instrumento, calculou-se o índice de concordância intra-avaliadores, o índice Kappa, e foram realizadas as correções necessárias. Com base nas respostas dos bailarinos que compuseram um banco de dados, procedeu-se a análise da estrutura interna. Inicialmente, o número de fatores a serem mantidos na análise fatorial exploratória foi verificado com base na análise paralela (Hayton, Allen \& Scarpello, 2004; Watkins, 2006). Para realizar a análise, foi utilizado o software livre "R", versão 2.15.3, por este possibilitar o uso da análise paralela para variáveis policóricas, via pacote psych (Revelle, 2018).

A partir disso, foi gerado um banco de dados para o software MPlus, versão 6.12 (Muthén \& Muthén, 1998-2011), para realizar a Exploratory Structural Equation Modeling (E-SEM), que também apresenta índices de ajuste que indicam a adequação da estrutura encontrada com base na presente amostra. Ressalta-se, ainda, que o ajustamento nos índices confirmatórios também sugere a adequação da amostra para a realização da análise. Considerando os fatores encontrados, foram investigados os coeficientes de consistência interna pelo alfa de Cronbach. 


\section{Resultados}

\section{Evidências Baseadas no Conteúdo}

Para iniciar a aplicação do instrumento em bailarinos, algumas adaptações no instrumento para o contexto da dança foram realizadas. Poucas mudanças de terminologia foram necessárias. Substituiu-se o termo treinador por professor e quando utilizado o termo equipe técnica, acrescentou-se uma explicação entre parênteses, afirmando que seriam coreógrafo e ensaiador. Os termos treinamentos e competições foram substituídos por ensaios, aulas, festivais e espetáculos. Os juízes propuseram apenas uma alteração, como substituir o termo "treinamentos" por "ensaios/aulas", que foi acatada. Resguardou-se, portanto, as especificidades da dança, o que se não fosse feito, poderia comprometer a compreensão dos bailarinos.

A análise dos juízes apresentou uma concordância, através do índice Kappa, de 90\% para pertinência teórica, $83 \%$ para pertinência prática e $85 \%$ para linguagem (semântica e apropriação para a idade dos participantes), valores esses considerados excelentes (Landis \& Koch, 1977). Os juízes propuseram pequenas alterações, que foram acatadas. Em relação ao coeficiente de validade de conteúdo, obtiveram-se os seguintes resultados: para linguagem (0.94), pertinência prática (0.95) e pertinência teórica (0.95). Com esses resultados, considera-se que o Questionário sobre Qualidade de Vida de Atletas - adaptado para Bailarinos (QQVA-B) possui evidências de conteúdo suficientes para ser utilizado.

\section{Evidências Baseadas na Estrutura Interna}

Para verificação das propriedades psicométricas do QQVA-B, investigou-se a melhor solução fatorial para o conjunto de itens do instrumento. Para tanto, partiu-se dos dados empíricos, isto é via E-SEM. Como primeiro passo, foi estabelecido o número máximo de fatores a serem mantidos via análise paralela, sendo que este procedimento indicou até cinco fatores (Figura 1). A partir disso, forçou-se soluções de dois a cinco fatores, utilizando a rotação oblíqua geomin, e método de extração Maximum Likelihood Robust (MLR), sendo este apropriado para correlações policóricas. Ressalta-se que a solução composta por cinco fatores não convergiu, possivelmente por correlações insuficientes dado o número de fatores, por isso foi desconsiderada nas verificações sequentes.

Os índices de ajuste fornecidos por essa análise ( $\mathrm{X}^{2} / \mathrm{df}$, RMSEA, CFI e SMR) indicaram melhor adequação da solução composta por quatro fatores, de acordo com os critérios adotados (Hooper, Coughlan \& Mullen, 2008). A partir disso, verificou-se a interpretabilidade das soluções três soluções investigadas. A solução de quatro fatores apresentou a melhor interpretação para os conjuntos de itens. Os índices de ajuste para essa solução foram $\mathrm{X}^{2} /$ $\mathrm{df}=2.02$; $\mathrm{RMSEA}=0.059 ; \mathrm{CFI}=0.960 ; \mathrm{e}, \mathrm{SMR}=$ 0.031. Considerando os pontos de corte (Hooper, Coughlan, \& Mullen, 2008), os índices de ajuste foram bons ( $\mathrm{X}^{2} / \mathrm{df}$, CFI e SMR) e aceitáveis (RMSEA). São apresentados na Tabela 1, as cargas fatoriais encontradas, o número de itens mantidos por fator e os índices de consistência interna (coeficiente alfa). O QQVA-B ficou, assim, 12 itens, agrupados em quatro fatores (o Fator 5 - Estado Emocional do Bailarino, com $\alpha=0.845$, foi eliminado):

Fator 1 - Relacionamento no Ambiente Esportivo $(\alpha=0.73)$, com 3 itens;

Fator 2 - Condições Básicas para a Saúde $(\alpha=0.74)$, com 3 itens;

Fator 3 - Sinais e Sintomas de Supertreinamento $(\alpha=0.83)$, com 4 itens;

Fator 4 - Planejamento e Periodização do Treinamento na Dança $(\alpha=0.53)$, com 2.

Vê-se na Tabela 1, que apenas dois itens não entraram nos critérios adotados e, por isso, não foram mantidos em nenhum dos quatro fatores (estão destacados em cor cinza); além disso, o 
Eligen values of tetrachoric/polychoric matrix

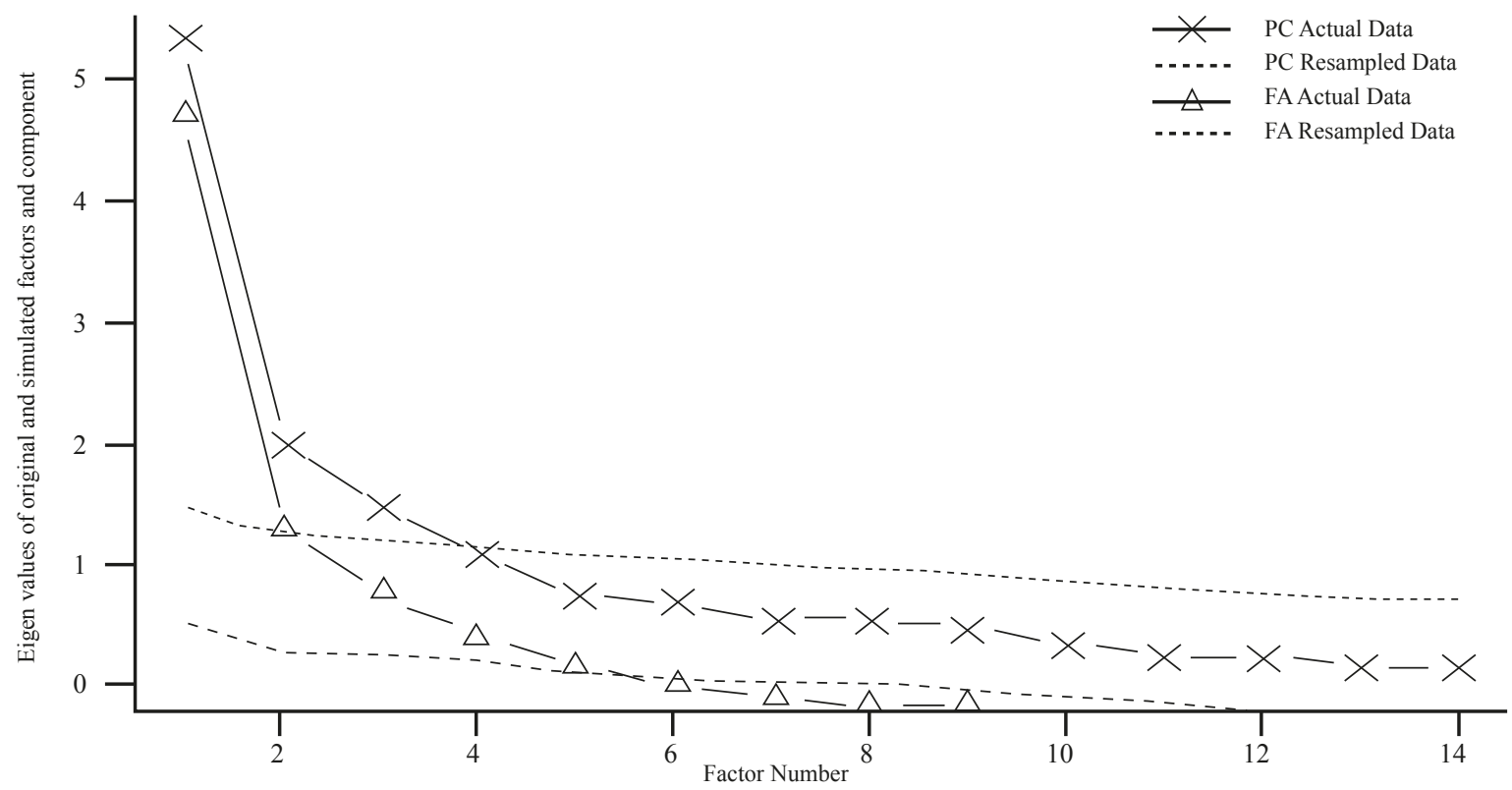

Figura 1. Apresentação gráfica da análise paralela

Tabela 1.

Análise fatorial exploratória e índices de consistência interna do Questionário de Qualidade de Vida sobre Atletas adaptado para Bailarinos $(Q Q V A-B)(N=313)$

\begin{tabular}{|c|c|c|c|c|}
\hline Item & Fator 1 & Fator 2 & Fator 3 & Fator 4 \\
\hline 1 & 0.60 & 0.15 & 0.17 & 0.10 \\
\hline 2 & 0.84 & 0.40 & 0.23 & 0.04 \\
\hline 3 & 0.74 & 0.40 & 0.26 & 0.13 \\
\hline 4 & 0.41 & 0.85 & 0.23 & 0.10 \\
\hline 5 & 0.34 & 0.70 & 0.41 & 0.17 \\
\hline 6 & 0.39 & 0.52 & 0.37 & 0.11 \\
\hline 7 & 0.29 & 0.27 & 0.83 & 0.30 \\
\hline 8 & 0.22 & 0.22 & 0.83 & 0.43 \\
\hline 9 & 0.25 & 0.20 & 0.67 & 0.31 \\
\hline 10 & 0.18 & 0.24 & 0.57 & 0.23 \\
\hline 11 & 0.39 & 0.24 & 0.42 & 0.20 \\
\hline 12 & 0.33 & 0.18 & 0.30 & 0.37 \\
\hline 13 & 0.11 & 0.12 & 0.40 & 0.90 \\
\hline 14 & 0.16 & 0.04 & 0.36 & 0.63 \\
\hline $\mathrm{N}^{o}$ de itens & 3 & 3 & 4 & 2 \\
\hline$\alpha$ & 0.73 & 0.74 & 0.83 & 0.84 \\
\hline
\end{tabular}


número de itens por fator variou entre dois e quatro. Alguns critérios foram utilizados para a manutenção dos itens na composição de cada fator. Consideramos apenas os itens com carga fatorial acima de 0.30 , que não prejudicassem ou não favorecessem a consistência interna do fator e o conteúdo interpretativo do item. A consistência interna para o conjunto total de 12 itens foi $\alpha=$ 0.82 , e a consistência interna dos quatro fatores variou entre 0.73 e 0.84 . Também se verificou a relação entre os fatores, que variou entre $r_{f l x f 4}=$ $0.14(p<0.05)$ e $r_{f 3 x f 4}=0.43(p<0.01)$.

Na sequência, para comparação entre a solução de quatro fatores e o modelo teórico, , buscando verificar qual dos modelos se ajustaria melhor aos dados, foi utilizada a análise fatorial confirmatória. Observa-se na Tabela 2 que as cargas fatoriais variaram entre 0.53 e 0.84 , e todos os itens apresentaram carga positiva. $\mathrm{O}$ erro encontrado foi inferior a 0.10 para todos os casos. Os índices de ajuste para o modelo confirmatório de cinco fatores foram $\mathrm{X}^{2} / \mathrm{df}=2.37$; RMSEA $=0.069 ; \mathrm{CFI}=0.905$; e $\mathrm{SMR}=0.065$. Esses índices são considerados, de modo geral, como bons ou aceitáveis, e bastante similares ao modelo empiricamente determinado. Os coeficientes de consistência interna foram iguais para quatro dos cinco fatores investigados na análise confirmatória, uma vez que esses quatro fatores apresentam a mesma composição em relação aos fatores da E-SEM; para o quarto fator do

Tabela 2.

Análise fatorial confirmatória do QQVA-B e fidedignidade por consistência interna $(n=313)$

\begin{tabular}{|c|c|c|c|c|}
\hline Fatores/Itens & Cargas & Erro & p-valor & $\begin{array}{l}\text { Consistência interna } \\
\qquad(\alpha)\end{array}$ \\
\hline \multicolumn{5}{|c|}{ F1- Relacionamento no Ambiente Esportivo } \\
\hline 1 & 0.57 & 0.05 & $<0.01$ & \multirow{3}{*}{0.73} \\
\hline 2 & 0.83 & 0.04 & $<0.01$ & \\
\hline 3 & 0.75 & 0.05 & $<0.01$ & \\
\hline \multicolumn{5}{|c|}{ F2 - Condições Básicas para a Saúde } \\
\hline 4 & 0.73 & 0.04 & $<0.01$ & \multirow{3}{*}{0.74} \\
\hline 5 & 0.77 & 0.04 & $<0.01$ & \\
\hline 6 & 0.59 & 0.05 & $<0.01$ & \\
\hline \multicolumn{5}{|c|}{ F3 - Sinais e Sintomas de Supertreinamento } \\
\hline 7 & 0.81 & 0.03 & $<0.01$ & \multirow{4}{*}{0.83} \\
\hline 8 & 0.82 & 0.03 & $<0.01$ & \\
\hline 9 & 0.68 & 0.04 & $<0.01$ & \\
\hline 10 & 0.59 & 0.05 & $<0.01$ & \\
\hline \multicolumn{5}{|c|}{ F4 - Planejamento e Periodização do Treinamento na Dança } \\
\hline 11 & 0.62 & 0.07 & $<0.01$ & \multirow{2}{*}{0.53} \\
\hline 12 & 0.54 & 0.06 & $<0.01$ & \\
\hline \multicolumn{5}{|c|}{ F5 - Estado Emocional do Bailarino } \\
\hline 13 & 0.86 & 0.07 & $<0.01$ & \multirow{2}{*}{0.84} \\
\hline 14 & 0.74 & 0.07 & $<0.01$ & \\
\hline
\end{tabular}


modelo confirmatório, não encontrado na E-SEM, obteve-se $\alpha=0.53$.

\section{Discussão}

Para melhor adequar a utilização de instrumentos psicológicos para bailarinos, este estudo procurou verificar as evidências de validade do QQVA (Cunha, 2008), propondo-se agora o QQVA-B. .De modo geral, os valores de alfa de Cronbach foram altos $(\alpha=0.73$ até $\alpha=0.84)$, garantindo a precisão do instrumento. Ao analisar o valor de alfa separadamente em cada fator, percebe-se que o maior valor está presente no Fator 5 - Estado Emocional do Bailarino $(\alpha=0.845)$, seguido do Fator 3 - Sinais e Sintomas de Supertreinamento $(\alpha=0.833)$. Esses valores indicam que os fatores com maiores magnitudes de efeito para a qualidade de vida dos bailarinos referem-se a aspectos emocionais e ao supertreinamento. Contudo, diferem da validação original do instrumento de Cunha (2008), em que os maiores valores foram encontrados para o Fator 1 - Relacionamento no Ambiente Esportivo $(\alpha=$ 0.855), seguido do Fator 2 - Condições Básicas para a Saúde $(\alpha=0.820)$, confirmando assim a necessidade da busca de evidências de validade, considerando as especificidades da modalidade de prática física (Gonçalves \& Belo, 2007).

Outra diferença na validação para bailarinos em relação àquela feita para atletas ocorreu no alfa de Cronbach do Fator 4 - Planejamento e Periodização do Treinamento na Dança $(\alpha=0.532)$-, com valor considerado abaixo do satisfatório (Landis \& Koch, 1977) e inferior ao proposto originalmente, no qual se obteve um valor maior $(\alpha=0.793)$. Optou-se por manter essa dimensão no QQVA-B pela possibilidade de um erro amostral, mas, sugere-se a aplicação do QQVA-B em outra população de bailarinos a fim de verificar a precisão (Clark \& Watson, 1995). Entretanto, deve-se considerar que esse fator foi composto por somente dois itens, o que pode ter impacto o coeficiente de consistência interna observado.
Além disso, nesse estudo utilizaram-se métodos de análise mais robustos, o que acaba se tornando um limitador o fato de o fator possuir apenas 2 itens.

De qualquer maneira, a baixa precisão deste fator significa que ele não explica significativamente a qualidade de vida de bailarinos. E, segundo Silva e Tkac (2012), os bailarinos não possuem um sistema de treinamento tão padronizado e controlado quanto os atletas, o que pode ter resultado na falta de expressividade deste fator.

\section{Considerações Finais}

Ressalta-se a importância do estudo da qualidade de vida de bailarinos, um aspecto que engloba algumas variáveis e que pode ser prejudicado pela intensidade dos treinamentos, afetando os domínios físico, psicológico, social, entre outros. É necessário que tenham questionários apropriados para essa população, a fim de mensurar esse e outros construtos que influenciam no bem-estar e na saúde dos dançarinos.

O instrumento QQVA-B é adequado para utilização em bailarinos adolescentes por possuir propriedades psicométricas satisfatórias. Entretanto, o Fator 4 - Planejamento e Periodização do Treinamento na Dança - precisa ser avaliado com cautela, pois apresentou baixa precisão. Novos estudos com outros bailarinos precisam ser realizados a fim de se verificar se a baixa precisão deste fator se mantém ou se ela ocorreu devido, apenas, a um erro amostral.

É relevante destacar que esta pesquisa se limita às evidências de validade de conteúdo e de consistência interna do instrumento. Sugerem-se, portanto, novos estudos, para que sejam verificadas as evidências de validade baseadas no processo de resposta, nas relações com variáveis externas e nas consequências da testagem. Só assim poder-se-á ter a real dimensão e aplicabilidade do instrumento, bem como realizar a normatização e padronização do instrumento. 
Ressalta-se, também, a importância da validação de outros instrumentos psicológicos para bailarinos, tendo em vista a carência desse procedimento voltado para essa população. Com essa análise, foi possível perceber que existem diferenças entre atletas e bailarinos a ponto de alterar a magnitude de efeito de fatores de um instrumento, dependendo da modalidade em questão.

\section{Referências}

Akranaviciute, D., \& Ruzevicius, J. (2007). Quality of life and its components: measurement. Engineering Economics, 52(2), 44-49.

Aksoy, M. C., \& Aksoy D. Y. (2015). Physical abuse of adolescent and young athletes. In M. N. Dord, R. N. Tandogan, G. Mann, \& R. Verdonk. (Eds.). Sports injuries: prevention, diagnosis, treatment and rehabilitation. ( $2^{\mathrm{a}}$ ed. pp. 26352642). New York: Springer.

Balbinotti, M. A., Benetti, C., \& Terra, P. R. S. (2007). Translation and validation of the Graham-Harvey survey for the Brazilian context. International Journal of Managerial Finance, 3(1), 26-48.

Breinbauer, C., \& Maddaleno, M. (2008). Nova abordagem para classificar os estágios de desenvolvimento do adolescente. In C. Breinbauer, \& M. Maddaleno (Eds.), Jovens: escolhas de mudanças: promovendo comportamentos saudáveis (pp. 212-221). São Paulo: Roca.

Clark, L. A., \& Watson, D. (1995). Constructing validity: Basic issues in objective scale development. Psychological Assessment, 7(3), 309319. Recuperado de: http://www.personal.kent. edu/ dfresco/CRM_Readings/Clark_and_Watson_1995.pdf

Cunha, R. A. (2008). Elaboração e validação do Questionário sobre Qualidade de Vida de Atletas (QQVA). (Dissertação de Mestrado não publicada, Departamento de Educação Física, Universidade Federal de Minas Gerais, Belo Horizonte, MG).
Esteves, A. M., Silva, A., Barreto, A., Cavagneli, D. A., Ortega, L. S., Parsons, A., ... Mello, M. T. (2015). Avaliação da qualidade de vida e do sono de atletas paralímpicos brasileiros. Revista Brasileira de Medicina do Esporte, 21(1), 53-56.

Fayers, P., \& Machin, D. (2013). Quality of life: the assessment, analysis, and interpretation of patient-reported outcomes. San Francisco: Wiley Editorial.

Gonçalves, M. P., \& Belo, R. P. (2007). Ansiedade-traço competitiva: diferenças quanto ao gênero, faixa etária, experiência em competições e modalidade esportiva em jovens atletas. $P s i$ co-USF, 12(2), 301-307.

Hayton, J. C., Allen, D. G., \& Scarpello, V. (2004). Factor retention decisions in exploratory factor analysis: A tutorial on parallel analysis. Organizational Research Methods, 7(2), 191-207. Doi: 10.1177/1094428104263675

Hernandéz-Nieto, R. A. (2002). Contributions to statistical analysis. Mérida: Universidad de Los Andes.

Hooper, D., Coughlan, J., \& Mullen, M. R. (2008). Structural Equation Modelling: Guidelines for Determining Model Fit. The Electronic Journal of Business Research Methods, 6(1), 53-60. Recuperado de: http://www.scirp.org/(S(i43dyn45teexjx455qlt3d2q))/reference/ReferencesPapers.aspx ?ReferenceID $=1617449$. Acesso em: 24 de outubro de 2018.

Landis, J. R., \& Koch, G. G. (1977). The measurement of observer agreement for categorical data. Biometrics, 33(1), 159-174. Doi: 10.2307/2529310

Lima, K. A., Silva, P. H. B., \& Barreto, R. R. (2014). Características das lesões em bailarinos e sua relação com a qualidade de vida. Revista Movimenta, 7(1).

Matsudo, S. M., Matsudo, V. R., Araújo, T., Andrade, D., Andrade, E., Oliveira, L., \& Braggion, G. (2002). Nível de atividade física da população do Estado de São Paulo: análise de acordo com o gênero, idade, nível sócio-econômico, distri- 
buição geográfica e de conhecimento. Revista Brasileira de Ciência e Movimento, 10(4), 41-50.

Muthén, L. K., \& Muthén, B. O. (1998-2011). Mplus user's guide. Sixth Edition. Los Angeles, CA: Muthén \& Muthén.

Pasquali, L. (2004). Psicometria: teoria dos testes na psicologia e na educação. Petrópolis: Vozes.

Revelle, W. (2018). Package “psych”. CRAN. Recuperado de https://cran.r-project.org/web/packages/psych/psych.pdf

Silva, A. M. B., \& Tkac, C. M. (2012). Relação entre força de membros inferiores e desempenho em bailarinos. Revista Brasileira do Movimento Humano, 2(3), 42-51.

Silva, A. M. B., Luz, T. S. R., Afonso, R. M, Araújo, M. F., Bittencourt, I. G., Carvalho, L. F., \& Enumo, S. R. F. (2015). Escala de Autoeficácia para Bailarinos (AEBAI): Construção e evidências de validade. Avaliação Psicológica, 14(1), 83-88.
Silveira, G. C. F., \& Pinto, J. F. (2001). Educação Física na perspectiva da cultura corporal: Uma proposta pedagógica. Revista Brasileira de Ciências do Esporte, 22(3), 137-150.

Watkins, M. W. (2006). Determining parallel analysis criteria. Journal of Modern Applied Statistical Methods, 5(2), 344-346. Doi: 10.22237/ jmasm/1162354020

World Health Organization. (1997). Life skills education for children and adolescents in schools. Geneva: Programme on Mental Health World Health Organization.

World Health Organization Quality of Life Group (WHOQOL). (1994). The development of the World Health Organization Quality of Life assessment instrument (the WHOQOL). In J. Orley, \& W. Kuyken (Eds.), Quality of life assessment: International perspectives (pp. 4160). Heigelberg: Springer Verlag.

\section{Recebido: 10 de fevereiro de 2016 Aprovado: 19 de setembro de 2018}


